\title{
AN INSIGHT INTO CONTEMPORARY TREND IN THE ANTIBIOGRAM OF SALMONELLA ENTERICA SEROVARS: A CLAMOR FOR ANTIBIOTIC RECYCLING.
}

Shaik Mohammed Usman'1, Rashmi M²

\section{HOW TO CITE THIS ARTICLE:}

Shaik Mohammed Usman, Rashmi M. "An insight into contemporary trend in the antibiogram of salmonella enterica serovars: a clamor for antibiotic recycling". Journal of Evolution of Medical and Dental Sciences 2013; Vol. 2, Issue 44, November 04; Page: 8468-8474.

ABSTRACT: CONTEXT: Enteric fever prevails to be a considerable health issue worldwide. Ciprofloxacin became the mainstay of its therapy after multidrug resistant salmonellae emerged. Treatment failure was soon recorded with ciprofloxacin, the index being in-vitro resistance to nalidixic acid. Chloramphenicol sensitive strains have resurfaced. Resistance to alternativesmacrolides is cropping up though not documented widely. These variations in addition to the fact that India is witnessing escalating resistance to antibiotics, stress the significance of continual surveillance of salmonellae resistance. INTENT: The study undertaken intends to document the current deviations in the antibiogram of the Salmonella isolates from blood culture, with a perspective of evaluating ciprofloxacin susceptibility and macrolide resistance, further considering, if there is a need to modify the antibiotic strategies for this condition. METHODOLOGY: The isolates from blood cultures from January 2012 to September 2013 obtained using commercial BACTEC 9120 system, sub-cultured and processed for determining salmonellae serotypes were further subjected to drug susceptibility testing for patterning their resistance. MICs to ciprofloxacin of nalidixic acid resistant (NAR) isolates were recognized. RESULTS: Salmonella was isolated from 114 out of 8210 blood culture specimens received. There were 74 Salmonella Typhi isolates, 39 Salmonella Paratyphi A and 1 Salmonella Paratyphi B. Highest resistance of salmonellae (85.96\%) was exhibited to nalidixic acid and highest susceptibility (100\%) to ceftriaxone. Decreased ciprofloxacin susceptibility-DCS (MIC of $0.125-1 \mu \mathrm{g} / \mathrm{ml}$ ) was noticed in around $90 \%$ of NAR salmonellae. Isolates demonstrated sizable resistance (42.10\%) to azithromycin. Only three strains were multidrug resistant. INTERPRETATION AND CONCLUSION: Routine screening of nalidixic acid sensitivity should be encouraged as a means to predict decreased fluoroquinolone susceptibility, thereby preventing therapeutic failure. In order to avert the profound impact of antimicrobial resistance and to define the best possible therapy, periodic monitoring of Salmonella antibiogram is of prime value. The upsurge of occurrence of ciprofloxacin resistance, impending resistance to macrolide- azithromycin, re-emergence of chloramphenicol sensitive strains is of particular concern and necessitates a change towards 'evidence-based' treatment and rationalization of existing empirical therapy of enteric fever shifting the vital focus towards antibiotic recycling.

KEY WORDS: Salmonella Typhi, Salmonella Paratyphi A, nalidixic acid, ciprofloxacin, azithromycin, minimum inhibitory concentration.

BACKGROUND AND LITERATURE REVIEW: Enteric fever, a common cause of PUO, is a global health concern and especially in endemic regions like India due to poor sanitation and personal hygiene. It is a major infectious disease seen with high fluctuating incidences. The World Health 
Organization (WHO) has on a global scale estimated that annually typhoid fever accounts for 21.7 million illnesses (217,000 deaths) and paratyphoid fever causes 5.4 million of these cases.[1] Highest incidence of enteric fever ( $>100$ cases per 100,000 persons per year) is witnessed at South Central Asia and Southeast Asia. An Indian study based at Delhi marked the incidence of enteric fever to be 9.8 cases per 1,000 person-years. [2] Enteric fever is usually caused by S. enterica subsp. enterica serovars Typhi and Paratyphi A, serovar Typhi being the commonest in India.[3]

Since its introduction in 1948, chloramphenicol has been the drug of choice for typhoid fever and remained the standard against which newer antimicrobials must be compared. Due to its association with the emergence of resistance, a high relapse rate, a high rate of continued and chronic carriage, bone marrow toxicity; ampicillin and co-trimoxazole were considered as the alternative antibiotics. Ampicillin and amoxicillin have been the treatment of choice in pregnancy and neonates. ${ }^{[4]}$ Resistance to all first line antimicrobials- ampicillin, trimethoprim-sulfamethoxazole and chloramphenicol is defined as multidrug resistance (MDR).[5]

Since the widespread resistance to chloramphenicol, ciprofloxacin has turned into the firstline drug of choice. Increased use of ciprofloxacin not just in clinical practise but also in veterinary medicine has resulted in the rapid emergence of Salmonella enterica serovars with reduced susceptibility to ciprofloxacin due to a point mutation in the gyr A gene, at either Ser-83 or Asp87.[6,7] These strains are associated with treatment failure of or a delayed response (prolonged fever clearance times) to ciprofloxacin therapy.[8,9] Reports on treatment failure after administration of ciprofloxacin to patients with enteric fever are increasing in India too.[10] Treatment failures have been defined in strains displaying decreased ciprofloxacin susceptibility (DCS) [Ciprofloxacin Minimum inhibitory concentration (MIC) of $0.125-1.0 \mu \mathrm{g} / \mathrm{ml}]$. [11] These strains are still considered conventionally susceptible to ciprofloxacin based on their Clinical Laboratory Standards Institute (CLSI) cut-off[12], but are resistant to nalidixic acid and thus are termed nalidixic acid-resistant S. enterica serovars.

According to various studies, the best predictor for DCS is nalidixic acid resistance (NAR). Ray et al[13] found that nalidixic acid susceptibility was a good marker for fluoroquinolone susceptibility but NAR had a poor predictive value for ciprofloxacin resistance and suggested that NAR isolates should be tested for ciprofloxacin MIC before deciding a change in therapeutic regimen. Patients with NAR strains require a higher dose of ciprofloxacin $(10 \mathrm{mg} / \mathrm{kg}$ twice daily for 10 days $)$ or ofloxacin (10-15 mg/kg divided twice daily for 7-10 days).[14]

Extended - spectrum cephalosporins and azithromycin are the other options available for the treatment of enteric fever. Azithromycin, a macrolide antibiotic, has been used as an alternative drug. It achieves low intravascular levels, has high intracellular tissue penetration, and a long elimination half-life of 72 hours. These properties make for once-daily administration and reduction in the duration of therapy. The drug is rapidly absorbed from the gut and is well-tolerated when used orally. However, there are reports of emergence of resistance to azithromycin.[15]

These inconstancies in the sensitivity patterns incited us to consider this study to document the variations in the antibiotic susceptibility of the Salmonella isolates from blood culture, to ultimately aid in the formulation of better strategies.

MATERIALS AND METHODS: This prospective study was executed at M S Ramaiah Memorial hospital, a tertiary care set up at Bangalore from January 2012 to September 2013. During this period of 21 months, 8210 blood samples from both in-patients and out-patients were received for 
aerobic bacterial culture. Blood culture was done in a commercial BACTEC Aerobic Plus blood culture (BACTEC 9120 system, Becton and Dickinson, U.S.A) and BACTEC bottles were processed as per manufacturer's recommendations. The cultures were incubated at $37^{\circ} \mathrm{C}$ for five days before declaring negative. BACTEC cultures were subcultured on Blood agar and MacConkey's agar, only after the machine flagged positive. The isolates producing characteristic colonies were identified by conventional biochemical tests and confirmed by agglutination with Salmonella antisera (Denka Seiken Co. Pvt. Ltd, Niigata, Japan).[16]

Salmonella isolates were subjected to antibiotic susceptibility test by Kirby-Bauer's disc diffusion method and interpreted as per Clinical laboratory Standards Institute (CLSI) guidelines[17] using Commercially available disks (Himedia Laboratories, Mumbai) of Ampicillin, Azithromycin, Cefixime, Cefoxitin, Ceftriaxone, Chloramphenicol, Ciprofloxacin, Cotrimoxazole, Furazolidone and Nalidixic acid. MICs to ciprofloxacin of NAR isolates were determined by E-test strips: Himedia Ezy МICтм Strips (Himedia Laboratories, Mumbai) with dilution ranging from $0.002-32 \mu \mathrm{g} / \mathrm{ml}$. Resistance to ampicillin, chloramphenicol and co-trimoxazole was entitled as MDR.

RESULTS: A total of 114 salmonellae were isolated from 8210 samples included in the study, indicating an incidence rate of $1.39 \%$. S. Typhi accounted for $64.91 \%$ (74/114) of the total isolates and S. Paratyphi A-34.21\% (39/114). Only one isolate of S. Paratyphi B was obtained. Majority of the positive samples were from males (78/114) and most of the positive cases belonged to age group of 21-30 years [Table-1].

Nearly $3 \%(\mathrm{~N}=3)$ of the isolates were found to be MDR. None of the salmonellae were resistant to ceftriaxone. Surprisingly, we discovered azithromycin resistance in around $42 \%$ of the isolates. High est resistance (85.96\%) was observed against nalidixic acid [Table-2]. Only 6 (5.26\%) of the total isolates were resistant to ciprofloxacin and also exhibited NAR showing MICs to ciprofloxacin of $>1 \mu \mathrm{g} / \mathrm{ml}$. The isolates showed very low-level resistance against the the remaining antibiotics- Ampicillin (2.63\%), Cefixime (0.88\%), Cefoxitin (2.63\%), Chloramphenicol (3.51\%), Cotrimoxazole (2.63\%) and Furazolidone (0.88\%).

Majority of the NAR isolates (89.79\%) showed MICs to ciprofloxacin of $0.125-1 \mu \mathrm{g} / \mathrm{ml}$ indicating DCS. MICs of $<0.125$ was seen in an NAR isolate, each of S. Typhi and S. Paratyphi A accounting to an insignificant proportion [Table-3].

\begin{tabular}{|c|l|l|l|}
\hline Age (years) & Male/s - N (\%) & Female/s- N (\%) & Total- N (\%) \\
\hline $0-10$ & $15(13.16)$ & $10(8.77)$ & $25(21.92)$ \\
$11-20$ & $16(14.04)$ & $9(7.9)$ & $25(21.92)$ \\
$21-30$ & $35(30.7)$ & $8(7.01)$ & $43(37.71)$ \\
$31-40$ & $10(8.77)$ & $3(2.63)$ & $13(11.4)$ \\
$41-50$ & $1(0.87)$ & $3(2.63)$ & $4(3.5)$ \\
$51-60$ & $0(0)$ & $1(0.87)$ & $1(0.87)$ \\
$61-70$ & $0(0)$ & $1(0.87)$ & $1(0.87)$ \\
$71-80$ & $1(0.87)$ & $0(0)$ & $1(0.87)$ \\
$81-90$ & $0(0)$ & $1(0.87)$ & $1(0.87)$ \\
Total & $78(68.42)$ & $36(31.58)$ & $114(100)$ \\
\hline \multicolumn{3}{|c|}{ Table-1: Age and gender wise distribution of } \\
\hline \multicolumn{3}{|c|}{ patien ts with positive Salmonella culture }
\end{tabular}




\begin{tabular}{|c|c|c|c|c|}
\hline \multirow[b]{2}{*}{ ANTIBIOTIC } & \multicolumn{3}{|c|}{ No. of isolates sensitive (\%) } & \multirow{2}{*}{$\begin{array}{c}\text { TOTAL- No. }(\%) \\
(\mathrm{N}=114)\end{array}$} \\
\hline & $\begin{array}{l}\text { S. Typhi } \\
(\mathrm{N}=74)\end{array}$ & $\begin{array}{l}\text { S. Paratyphi A } \\
\qquad(\mathrm{N}=39)\end{array}$ & $\begin{array}{c}\text { S. Paratyphi B } \\
(\mathrm{N}=1)\end{array}$ & \\
\hline Ampicillin & $71(95.95)$ & $39(100)$ & $1(100)$ & 111 (97.37) \\
\hline Azithromycin & $45(60.80)$ & $21(53.85)$ & $0(0)$ & $66(57.9)$ \\
\hline Cefixime & $74(100)$ & $38(97.44)$ & $1(100)$ & $113(99.12)$ \\
\hline Cefoxitin & $73(98.65)$ & 37 (94.87) & $1(100)$ & 111 (97.37) \\
\hline Ceftriaxone & $74(100)$ & $39(100)$ & $1(100)$ & $114(100)$ \\
\hline Chloramphenicol & $70(94.59)$ & $39(100)$ & $1(100)$ & $110(96.49)$ \\
\hline Ciprofloxacin & $69(93.24)$ & 37 (94.87) & $1(100)$ & $108(94.74)$ \\
\hline Cotrimoxazole & $71(95.95)$ & $39(100)$ & $1(100)$ & 111 (97.37) \\
\hline Furazolidone & $73(98.65)$ & $39(100)$ & $1(100)$ & $113(99.12)$ \\
\hline Nalidixic acid & $8(10.81)$ & $7(17.95)$ & $1(100)$ & $16(14.04)$ \\
\hline
\end{tabular}

\begin{tabular}{|c|c|c|c|c|c|c|c|c|c|c|c|c|}
\hline MIC $(\mu \mathrm{g} / \mathrm{ml})$ & $<0.125$ & 0.125 & 0.190 & 0.250 & 0.380 & 0.500 & 0.750 & 1 & 1.5 & 2 & 3 & 4 \\
\hline S. Typhi $(\mathrm{N}=66)$ & 1 & 5 & 6 & 8 & 7 & 14 & 9 & 11 & 2 & 2 & 0 & 1 \\
\hline S. Paratyphi A (N=32) & 1 & 2 & 0 & 2 & 3 & 6 & 7 & 8 & 0 & 1 & 1 & 1 \\
\hline
\end{tabular}

Table-3: MIC values of NAR salmonellae to ciprofloxacin

DISCUSSION: This study reveals high incidence of NAR strains which are associated with high MICs to ciprofloxacin, and this is in accordance with other studies, who found a high resistance to nalidixic acid ( $>90 \%$ ), documented MICs of $>0.5 \mu \mathrm{g} / \mathrm{ml}$ to ciprofloxacin in majority of their isolates. $[18,19]$

Due to rising incidence of NAR, a marker for predicting low-level resistance and treatment failure to ciprofloxacin[8-13], CLSI has now recommended that all S. Typhi isolates should be screened for NAR along with ciprofloxacin for optimal interpretation of susceptibility.[20] Any isolate that shows NAR should be reported as intermediately susceptible to ciprofloxacin.[21]

The data presented in our study appraises that MDR exists in an almost trivial manner. This finding is comparable with recent reports from some regions where the incidence of MDR isolates appeared to have decreased.[22, 23] The low frequency of MDR is remarkable, since these drugs could once again be used for the treatment. Discontinuation of chloramphenicol therapy relieved the selection pressure paving the way for re-emergence of sensitive strains to chloramphenicol, as shown here and been widely reported very recently from many other parts of India.[11, 24] This reappearance of sensitive strains to first line antimicrobials in previously resistant areas points towards the indispensable concept of antibiotic recycling, preserving the use of older antibiotics. [25] All the isolates were sensitive to ceftriaxone in our study which corresponds to the study of others.[26, 27]

The present communication also endorses that S. Paratyphi A is a emerging pathogen of enteric fever with an up-swinging incidence. We isolated one S.Paratyphi B, known to be very rare in India.[28] 
CONCLUSION: The rising MIC values of ciprofloxacin and impending resistance to macrolide azithromycin is alarming and of particular concern leading to prolonged treatment, delayed recovery, and post treatment failures. This may necessitate a change towards 'evidence-based' treatment. With wide reports of a remarkable decrease over the years in resistance to first line drugs, the policy of empirical treatment of enteric fever needs to be rationalized and demands reconsideration of first-line antibiotics as an effective component, asserting the indispensable concept of antibiotic recycling.

This study re-emphasizes the past and current problems encountered in the treatment of enteric fever, with an anticipation of new patterns of antimicrobial resistance in the foreseeable future. Wide variation in the sensitivity pattern of various salmonellae strains circulating in different geographic regions makes it near mandatory for constant surveillance of antimicrobial susceptibility before instituting therapy and caution should be exercised periodically to avert the profound impact of antimicrobial resistance.

\section{REFERENCES:}

1. Crump JA, Luby SP, Mintz ED. The global burden of typhoid fever. Bull World Health Organ 2004;82:346-53.

2. Sinha A, Sazawal S, Kumar R. Typhoid fever in children aged less than 5 years. Lancet 1999;354:734-737.

3. Pillai PK, Prakash K. Current status of drug resistance and phage types of Salmonella typhi in India. Indian J Med Res 1993;97:154-158.

4. Murray PR, Baron EJ, Pfaller MA, Tenover FC, Yolken RH, editors. Manual of clinical microbiology. 9th Ed. Washington DC: ASM Press. 2007;1:679-83.

5. Wain J, Kidgell C. The emergence of multidrug resistance to antimicrobial agents for the treatment of typhoid fever. Trans R Soc Trop Med Hyg 2004;98:423-30.

6. Phung Le, Ryo H, Nomura T. Specific gyrA mutation at codon 83 in nalidixic acid-resistant Salmonella enterica serovar Typhi strains isolated from Vietnamese patients. Antimicrob. Agents Chemother. 2002;46:2052-2053.

7. Slinger R, Desjardins M, McCarthy AE, Ramotar K, Jessamine P, Guibord C et al. Suboptimal clinical response to ciprofloxacin in patients with enteric fever due to Salmonella spp. with reduced fluoroquinolone susceptibility: a case series. BMC Infect. Dis. 2004;4:36.

8. Threlfall EJ, Ward LR, Skinner JA, Smith HR, Lacey S. Ciprofloxacin resistant Salmonella typhi and treatment failure. Lancet 1999;353:1590-1591.

9. Crump JA, Kretsinger K, Gay K, Hoekstra RM, Vugia DJ, Hurd S et al. Clinical response and outcome of infection with Salmonella enterica serotype Typhi with decreased susceptibility to fluoroquinolones: A United States Food Net multicenter retrospective cohort study. Antimicrob Agents Chemother 2008;52:1278-84.

10. Dhanashree B. Antibiotic susceptibility profile of Salmonella enterica serovars: Trend over three years showing re-emergence of Chloramphenicol sensitivity and rare serovars. Indian J Med Sci 2007;61:576-9.

11. Parry CM. Antimicrobial resistance in Salmonella enterica. Curr Opin Infect Dis 2003;16:46772.

12. Clinical and Laboratory Standards Institute (CLSI). 2006. Performance standards for 
antimicrobial susceptibility testing; 16th informational supplement. M100-S16, vol. 26, no. 3. CLSI, Wayne, Pa.

13. Ray P, Sharma J, Marak RS, Garg RK. Predictive efficacy of nalidixic acid resistance as a marker of fluoroquinolone resistance in Salmonella enterica var Typhi. Indian J Med Res. 2006; 124:105-8.

14. Miller IS, Pegues DA. Salmonella species, including Salmonella Typhi. In: Mandell GL, Bennett JE, Dolin R, editors. Mandell, Douglas and Bennetts principles and practice of infectious disease. 5th Ed. Philadelphia: Churchill Livingstone; 2000. p. 2344-63.

15. Reddy S, Rangaiah J, Addiman S, Wareham D, Wilson P, Sefton A. Epidemiology, antibiotic trends and the cost of enteric fever in East London, 2005-2010. Travel Med Infect Dis. 2011;9(4):206-12.

16. Barrow GI, Feltham RK, editors. Characters of Gram negative bacteria. In: Cowan and Steels Manual for Identification of Medical Bacteria. 3rd ed. Cambridge: Cambridge University Press; 2003. p. 94-164.

17. CLSI. Performance Standards for Antimicrobial Susceptibility Testing: Nineteenth Informational Supplement. CLSI Document M100-S19. Wayne PA: Clinical and Laboratory Standards Institute; 2009.

18. Madhulika. Current pattern in antimicrobial susceptibility of salmonella typhi isolated In Pondicherry. Ind J Med Res. 2004;120:111-114.

19. Rodrigues C, Shenai S, Mehta A. Enteric Fever in Mumbai, India: the Good News and the Bad News. Clini Infect Dis. 2003;535:536.

20. Wayne, PA. Clinical and Laboratory Standards Institute (2006) Clinical and Laboratory Standards Institution: Methods for Dilution Antimicrobial Susceptibility Tests for Bacteria that Grow aerobically. Approved standard, CLSI document M7-A7.

21. Harish BN, Madhulika U, Parija SC. Current Pattern in antimicrobial susceptibility of Salmonella typhi isolates in Pondicherry. Ind J Med Res 2004;120:111-4.

22. Kumar Y, Sharma A, Mani KR. Re-emergence of susceptibility to conventionally used drugs among strains of Salmonella of Salmonella Typhi in central west India. J Infect Dev Ctries 2011;5:227-30.

23. Capoor MR, Nair D. Quinolone and cephalosporin resistance in enteric fever. J Glob Infect Dis 2010;2:258-62.

24. Mandal S, Mandal MD, Pal NK. Reduced minimum inhibitory concentration of Chloramphenicol for salmonella enterica serovar typhi. Indian J Med Sci 2004;58:16-23.

25. Harish BN, Menezes GA. Preserving efficacy of chloramphenicol against typhoid fever in a tertiary care hospital, India. Regional Health Forum, WHO South-East Asia Region. 2011;15:926.

26. Bajracharya BL, Baral MR, Shakya S, Tuladhar P, Poudel M, Acharya B. Clinical profile and antibiotic response in typhoid fever. KUMJ 2006;4(13):25-9.

27. Tankhilwala SS. An unusually high occurrence of salmonella enterica serotype paratyphi A in patients with enteric fever. Ind J Med Res. 2003;117:10-12.

28. Ananthnarayan R, Paniker KJ. Enterobacteriaceae III: Salmonella. In: Ananthnarayan and Paniker's Textbook of Microbiology. 8th ed. Hyderabad: Universities Press (India) Private Limited; 2009. p. 288-301. 


\section{AUTHORS:}

1. Shaik Mohammed Usman

2. Rashmi M.

\section{PARTICULARS OF CONTRIBUTORS:}

1. Assistant Professor, Department of Clinical Microbiology, Kannur Medical College, Kannur, Kerala, India.

2. Consultant, Department of Microbiology, Metropolis Diagnostics, M.S. Ramaiah Memorial Hospital, Bangalore, Karnataka, India.

\section{NAME ADDRESS EMAIL ID OF THE} CORRESPONDING AUTHOR:

Dr. Rashmi. M., \#309, $1^{\text {st }}$ Mai n, $1^{\text {st }}$ Cross, Sri Draupatham ba Road, Karanji Katte, Kolar - 563101, Karnataka, India.

Email -rashmi_2284@yahoo.com mrashmi22@gmail.com

Date of Submission: 16/10/2013.

Date of Peer Review: 19/10/2013.

Date of Acceptance: 24/10/2013.

Date of Publishing: 29/10/2013 